Ophthalmologe 2009 $106: 773-774$

DOI 10.1007/s00347-009-1958-5

Online publiziert: 5. September 2009

(c) Springer Medizin Verlag 2009
F. Tost

Klinik und Poliklinik für Augenheilkunde, Universitätsklinikum Greifswald

\title{
Homemonitoring in der Augenheilkunde
}

\author{
Ophthalmologische Versorgungsforschung \\ als Voraussetzung einer \\ „Individualisierten Medizin“
}

Der Erhalt von Gesundheit und damit auch des Sehvermögens als wesentliche Grundlage für Kommunikation und Lebensqualität gilt im Zeitalter der Information als zunehmend wachsendes Bedürfnis von Individuum und Gesellschaft. Gleichzeitig sehen komplexe gesundheitsökonomische Analysen eine zunehmend kritische Kostenentwicklung im Gesundheitssystem. Dieses Argument wird häufig bemüht, wenn innovative Versorgungsformen wie Homemonitoringsysteme breitere Anwendung finden sollen. In Wirklichkeit hebt die makroökonomische Ursachenbewertung der Kostensteigerungen im Gesundheitssystem jedoch nicht die unmittelbaren Folgen des medizinisch-technischen Fortschritts als entscheidenden Faktor hervor. Vielmehr bewertet sie zu starke Partikularinteressen einzelner Gesundheitsakteure, Bürokratie und Ressourcenverschwendung sowie das unzureichende Gesundheitswissen von Patienten als relevante Problemkreise auf dem Gesundheitssektor. Homemonitoringsysteme könnten einen wichtigen Beitrag zur Fortentwicklung der ophthalmologischen Behandlungsmöglichkeiten leisten, wenn sie gezielt mit Unterstützung des Augenarztes durch den geschulten Patienten Anwendung finden. Gegenwärtig ist der Versorgungsgrad chronisch Augenkranker mit spezifischen Angeboten extrem gering, obwohl das Interesse bei einem Teil der uns anvertrauten $\mathrm{Pa}$ tienten durchaus vorhanden ist, wie zwei
Übersichtsarbeiten mit ganz unterschiedlicher Herangehensweise (papierbasiert oder medizingerätetechnisch orientiert) zum Leitthema „Homemonitoring“ aufzeigen. Im Beitrag von A. Cordes et al. wird über Erfahrungen mit einem papiergestützten Selbsttest für Patienten mit altersbedingter Makuladegeneration und dessen augenarztunterstützendes Begleitmanagement berichtet. Über die Möglichkeiten und Grenzen der Medizingerätetechnik bei Glaukompatienten informieren anhand konkreter Erkenntnisse aus einer Versorgungsforschungsstudie C. Jürgens et al. Die Handhabung der Medizingerätetechnik muss in optimaler Weise auf den Anwender abgestimmt sein. Hier zeigen alle aktuellen Konzepte noch Schwierigkeiten, welche jedoch beim gegenwärtigen Stand der Technik lösbar wären! Mit den technischen Möglichkeiten von Homemonitoringsystemen in der $\mathrm{Au}$ genheilkunde befasst sich der Beitrag von R. Großjohann. Seine naturwissenschaftlich basierte Analyse und Darstellung der Messtechnik am Beispiel der Tonometer und die daraus folgende Interpretation von Messwerten verschiedener Gerätesysteme sind für ein erfolgreiches Homemonitoring von hoher praktischer Bedeutung, sowohl in der Augenheilkunde und als auch für andere Fachgebiete. Beachtung sollte auch seine Feststellung finden, dass andere medizinische Fachgebiete in der Formulierung und Schaffung von Standards für die Netzwerkfähigkeit von
Medizingeräten und in der Standardisierung der Schnittstellen und des Datenaustausches unter ähnlichen gesundheitsökonomischen Bedingungen bereits weitgehend Verbindlichkeit erreicht haben. Hinsichtlich des Datenschutzes und Umgangs mit persönlichen Patienteninformationen beim Homemonitoring gelten für die gesamte Medizin weitreichende gesetzliche Regelungen, wie sie F. Heydenreich vorstellt. Erst mit der breiten praktischen Nutzung telemedizinischer Homemonitoringverfahren wird eine Fortentwicklung der Rechtssprechung - sofern notwendig - vonstatten gehen.

Alle Beiträge gemeinsam zeigen auf, dass die wesentlichen Vorraussetzungen für den Einsatz von Teleophthalmologie und Homemonitoring bereits heute bestehen (und von anderen Fachgebieten schon intensiver genutzt werden). Für die erfolgreiche Fortentwicklung der Augenheilkunde wird es daher auch entscheidend sein, diese innovativen Versorgungsformen aktiv in den Betreuungsprozess chronisch Augenkranker zu integrieren. Natürlich können Homemonitoringsysteme auf lange Sicht nicht für jeden Patienten das ideale Gesundheitsangebot darstellen. Im Bestreben nach einer individualisierten Medizin, welche den Patienten zentral in den Mittelpunkt rückt, werden sie aber einen relevanten Beitrag leisten können, um das Gesundheitswissen und die Selbstverantwortung von $\mathrm{Pa}$ tienten zu stärken. 
Das Homemonitoring unterliegt als Teil des medizinischen Betreuungsprozesses auch den Wechselbeziehungen zwischen Arzt, Patient und Gesellschaft. In diesem Spannungsfeld muss die $\mathrm{Au}$ genheilkunde neue Wege finden, wie die Greifswalder Medizinische Fakultät und deren Universitätsklinikum, die eine „Individualisierte Medizin“ zu ihrem Leitbild erhoben haben [1]. Aus dem Department Community Medicine stellt N. van den Berg das Modellprojekt der „arztentlastenden, gemeindenahen, e-health-gestützten, systemische Interventionen beherrschenden Schwester" (Akronym: AGNES) vor. Danach können assistierende Gesundheitsberufe bei Wahrung des Delegationsverfahrens durch den Arzt eine arztunterstützende Arbeit in der Allgemeinmedizin leisten. Zukünftige Projekte der Versorgungsforschung in der Augenheilkunde sollten Aufschluss bringen, ob dieses Konzept nicht auch für eine breitere Integration des Homemonitorings in die augenheilkundliche Regelversorgung geeignet wäre. Die ophthalmologische Versorgungsforschung ist daher ein relevantes Forschungsziel des Fachgebietes und muss als solche aus den Gründen benannt und gefördert werden, wie sie $\mathrm{N}$. Pfeiffer [2] treffend formuliert hat.

$\mathrm{Ihr}$

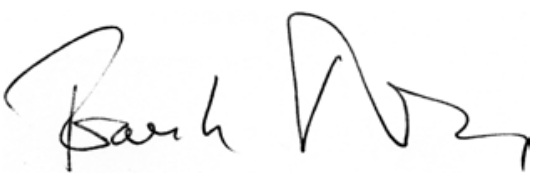

F. Tost

\section{Korrespondenzadresse}

Prof. Dr. F. Tost

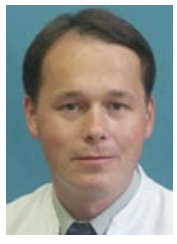

Klinik und Poliklinik für Augenheilkunde, Universitätsklinikum Greifswald Ferdinand-Sauerbruch-Straße, 17475 Greifswald Tost@uni-greifswald.de

Interessenkonflikt. Der korrespondierende Autor gibt an, dass kein Interessenkonflikt besteht.

\section{Literatur}

1. UKG - PI 38 v. 15.07.2009 Anspruch und Markenzeichen - die „Individualisierte Medizin“ wird in Greifswald zum Leitbild erhoben

2. Pfeiffer N (2008) Forschungsstandort deutschland. herausforderungen und ziele in der augenheilkunde. Ophthalmologe 105:1089

\section{Exklusiv für Abonnenten und Gesellschaftsmitglieder :}

\section{Nutzen Sie das Online-Archiv von Der Ophthalmologe}

Ihre Vorteile:

Komfortable und schnelle Recherche nach Themen, Autoren, Suchbegriffen

— Ob unterwegs oder am eigenen PC: Zugriff überall und jederzeit

- Online First: Lesen Sie die aktuellsten Beiträge schon vor Erscheinen des gedruckten Heftes online

\section{Registrieren Sie sich jetzt unter}

www.DerOphthalmologe.de

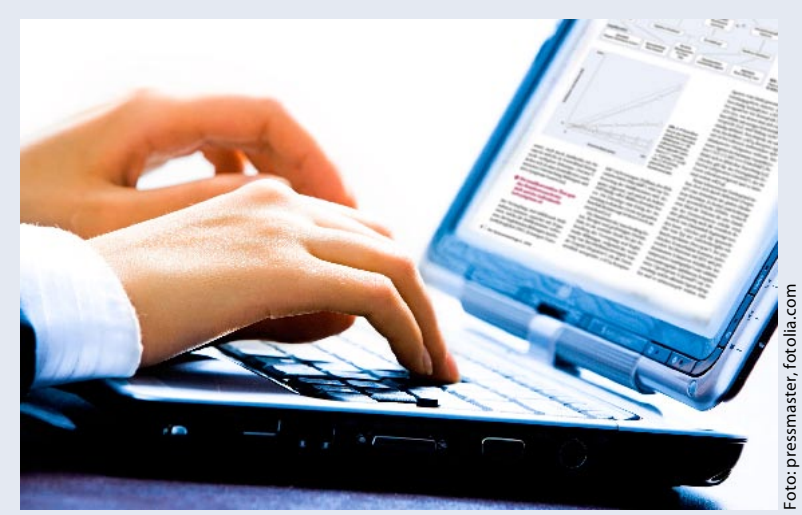

